\title{
PREPAROS DENTAIS COM FINALIDADE PROTÉTICA: UMA REVISÃO DA LITERATURA
}

\author{
TOOTH PREPARATION WITH PROSTHETIC PURPOSE: A LITERATURE REVIEW
}

\author{
Mônica Nogueira Pigozzo * \\ Dalva Cruz Laganá ** \\ Matsuyoshi Mori ${ }^{* * * *}$ \\ Carlos Gil ***** \\ Alessandra Galhardo Mantelli ******
}

\begin{abstract}
RESUMO
Diante da importância das próteses parciais fixas, próteses parciais removíveis e próteses híbridas, o principal objetivo deste trabalho é realizar uma revisão da literatura a respeito dos preparos dentais com finalidade protética. O preparo dental para uma prótese é um dos fatores críticos, que deve ser cuidadosamente planejado e executado para permitir a longevidade do tratamento. Esse deve restabelecer função, mastigatória e fonética, além de estética, requisitos estes fundamentais para a satisfação do paciente perante os resultados finais de uma reabilitaçáo. Em PPF a quantidade de desgaste das paredes dentais preparadas, angulação e localizaçáo do término cervical influenciam muito a longevidade e a estética da restauração final. O preparo dental, nesse tipo de prótese, deve permitir uma espessura adequada de material restaurador, ao mesmo tempo em que deve preservar a integridade pulpar e periodontal. Já nas PPRs o preparo de adequados planos-guias, descansos para apoios oclusais e contornos dentais se tornam extremamente necessários para o sucesso da reabilitação por meio desse tipo de prótese, que tem como objetivo a preservação da saúde bucal, conforto e função para o paciente. Já a redução da altura dos remanescentes radiculares em próteses híbridas é importante, pois diminui possíveis riscos de mobilidade e consequente perda do elemento, visto que a preservação dessas raízes previne perda óssea, promovendo melhora na retenção e estabilidade da prótese. Pelos trabalhos da literatura aqui revisados, pode-se concluir que, os preparos dentais são fatores primordiais no sucesso da reabilitação protética.
\end{abstract}

DESCRITORES: Preparo do dente • Próteses dentárias.

\section{ABSTRACT}

Considering the importance of fixed partial denture, removable partial denture and hybrid prosthesis, the main objective of this work is to realize a literature review about tooth preparation with prosthetic purpose. The prosthetic dental preparation is one of the critical factors that must be carefully planned and executed, to allow a longevity treatment. This must reestablish the phonetic and masticatory functions, phonetic and masticatory, function, beyond esthetics, and these requisites are basic for the patient's satisfaction with the final rehabilitation results. In PPD the quantity of wearing in the prepared dental walls, angulation and the margin location, influence the final restoration esthetics and longevity. The dental preparation, in this kind of prosthesis, must allow an adequate restoring material thickness, at the same time that the pulpal and periodontal integrity must be preserved. In the RPD the dental preparation of adequate guiding planes, occlusal rests and tooth contours become extremely necessary for the rehabilitation success in this kind of prosthesis, which present the objective to preserve the oral health, comfort and function for the patient. The root height reduction in hybrid prosthesis is important because it can diminishes possible risks of mobility and consequent loss of the dental element, because, the preservation of these roots prevents bone loss, improving the prosthesis retention and stability. Observing the papers revised here, it is concluded that tooth preparation is an important factor in the oral rehabilitation success.

DESCRIPTORS: Tooth preparation • Dental prosthesis.

\footnotetext{
* Doutoranda na área de Prótese Parcial Removível do Departamento de Prótese da Faculdade de Odontologia da Universidade de São Paulo - (USP). Email: mpigozzo@usp.br.

** Profa. Titular na área de Prótese Parcial Removível do Departamento de Prótese da Faculdade de Odontologia da Universidade de São Paulo- (USP).

*** Prof. Doutor na área de Prótese Fixa do Departamento de Prótese da Faculdade de Odontologia da Universidade de São Paulo- USP.

**** Prof. Titular na área de Prótese Parcial Removível do Departamento de Prótese da Faculdade de Odontologia da Universidade de São Paulo- (USP).

***** Mestranda na área de Prótese Parcial Removível do Departamento de Prótese da Faculdade de Odontologia da Universidade de São Paulo- (USP).
} 
Pigozzo MN, Laganá DC, Mori M, Gil C, Mantelli AG. Preparos dentais com finalidade protética: uma revisão da literatura. Revista de Odontologia da Universidade Cidade de São Paulo 2009 jan-abr; 21(1): 48-55

\section{INTRODUÇÃO}

As formas de retenção e a resistência de um preparo dental para uma prótese parcial fixa (PPF) são fatores críticos que devem ser cuidadosamente planejados e executados. Da mesma forma, o preparo dental para próteses parciais removíveis (PPR) ou próteses híbridas $\mathrm{(PH})$ também é crucial para garantir o sucesso da reabilitação.

As características do preparo dental para receber uma coroa, por exemplo, em metalocerâmica, têm sido relatadas constantemente na literatura e é universalmente aceito que a quantidade de desgaste das paredes dentais preparadas, angulação e localização do término cervical influenciam muito na longevidade e na estética da restauração final (Smith e Wilson ${ }^{35}$, 1998; Richter e Ueno $\left.{ }^{27}, 1973\right)$. O preparo dental em uma PPF deve permitir uma espessura adequada de material restaurador, ao mesmo tempo em que deve preservar a integridade pulpar. Essa espessura mínima requerida para a confecção da coroa depende da liga empregada. Porém, desgastes com espessura de 0,5 a $0,7 \mathrm{~mm}$ na vestibular, $1,5 \mathrm{~mm}$ na lingual e $2 \mathrm{~mm}$ na incisal, são aceitos como médias (Smith e Wilson ${ }^{35}$, 1998; Shillingburg e Hobo ${ }^{34}$, 1997; Hobo e Shilingburg ${ }^{16}$, 1973; Miller e Belsky ${ }^{20}$, 1977). Chandler $^{8}$ (1989) relatou que desgastes de $1,5 \mathrm{~mm}$ deixam o dente preparado com aproximadamente $25 \%$ da sua área inicial e uma espessura de dentina de apenas 0,5 $\mathrm{mm}$ até o órgão pulpar, deixando, dessa forma, pouco espaço para prováveis erros do operador. Assim, reduçóes excessivas levam à exposição da polpa e consequente perda da vitalidade dental, podendo resultar em um fracasso prematuro (Cheung et al. ${ }^{9}, 1990$ ).

Para a realização de uma reabilitação oral através de PPRs, o objetivo do profissional é confeccionar uma prótese que o paciente possa facilmente inserir e remover da boca, sem provocar danos aos elementos de suporte. Além disso, a prótese deve resistir à tendência de deslocamento provocado pelos alimentos e função mastigatória. Assim, o preparo de adequados planos-guias, descansos para apoios oclusais e contornos dentais se tornam extremamente necessários para o sucesso de casos utilizando esse tipo de prótese (Rudd et al. ${ }^{30}, 1999$ ). Esses preparos devem, dessa forma, permitir que os princípios básicos de abraçamento, retenção, estética, suporte e estabilidade sejam estabelecidos, promovendo a preservação da saúde bucal, conforto e função para o paciente.

Próteses híbridas promovem melhor função do que próteses totais convencionais, já que pacientes com esse tipo de prótese apresentam força de mastigação, eficiência mastigatória e controle nos movimentos mandibulares superior à pacientes desdentados. Além disso, a preservação de remanescentes dentários previne perda óssea, promovendo melhora na retenção e estabilidade da prótese. Para a confecção de próteses híbridas são necessários preparos desses remanescentes dentários, principalmente redução de suas alturas, diminuindo possível mobilidade e consequente perda do elemento.

Tendo em vista a importância das próteses parciais fixas, próteses parciais removíveis e próteses híbridas, o principal objetivo deste trabalho é realizar uma revisão da literatura a respeito dos Preparos dentais com finalidade protética.

\section{REVISÃO DA LITERATURA}

\section{Preparos dentais para PPFs}

Segundo Guyer ${ }^{14}$ (1970), as 4 etapas de preparo de um dente para receber uma PPF seria: (1) redução da incisal ou oclusal, (2) desgaste das paredes axiais, (3) término cervical e (4) forma de resistência e retenção. Segundo o mesmo autor, o melhor formato de desgaste oclusal é aquele que segue a anatomia dos dentes, pois permite uma adequada espessura de material restaurador, promove uma melhor distribuição de formas ao dente pilar, preserva a integridade pulpar, além de evitar o deslocamento da prótese. Em relação à redução axial também sedeve seguir a anatomia dental com convergência de $2^{\circ}$ a $5^{\circ}$ (aproximadamente 0,5 a $0,7 \mathrm{~mm}$ na vestibular, $1,5 \mathrm{~mm}$ na lingual e $2 \mathrm{~mm}$ na incisal). Em relação aos términos cervicais, o autor se classifica em: ângulo reto, ombro, ombro biselado e chamfrado. Para melhorar a resistência e retenção, o autor sugere a criaçáo de sulcos, caixas e uso de pinos.

Gardner ${ }^{12}$ (1982) relatou que o término cervical é um dos fatores mais importantes para garantir o sucesso de uma coroa. Existem basicamente 3 localizaçóes para este: supragengival, ao nível da crista gengival e subgengival. Lorato $^{18}$ (1969), Silness ${ }^{33}$ (1970) e Stein e Glickman ${ }^{37}$ (1960) apontaram, em um estudo, que grande parte de coroas com términos posicionados subgengivalmente apresenta alto índice de inflamação, diferentemente de coroas com términos supragengivais, que apresentaram gengiva com características saudáveis. Por outro lado, Goodacre et al. ${ }^{13}$ (2001) declararam que coroas estendidas para subgengival apresentam melhor estética, retenção, além de prevenir cáries na região cervical.

Em relaçâo ao tipo de término, Preston ${ }^{25}$ (1977) 
indica a utilização de chanfrado com ou sem bisel em coroas metálicas e em ombro com bisel em coroas com porcelana. Sozio ${ }^{36}$ (1977) recomenda a utilização de ombro com bisel para regióes estéticas e um ombro com chanfro em $45^{\circ}$ para metalocerâmicas. El-Ebrashi et al. ${ }^{11}$ (1969) realizaram um estudo experimental através de análise fotoelástica para avaliar a transmissão de forças em diferentes tipos de términos cervicais. Os autores relataram que o do tipo ombro com uma linha interna angulada (oblíqua) e o chanfrado foram os que apresentaram menor concentraçáo de forças, ao contrário, do ombro biselado que apresentou maior concentraçáo de forças. Guyer ${ }^{15}$ (1985) relatou que o uso do término cervical do tipo ombro com uma linha interna angulada (oblíqua) tendeu a desaparecer devido à dificuldade na sua obtenção, pois instrumentos rotatórios dificilmente conseguem definir seu formato, sendo sempre necessária a utilização de instrumentos manuais.

Ayad et al. ${ }^{2}$ (1996) realizaram um trabalho com o objetivo de avaliar a rugosidade superficial de preparos protéticos, utilizando para isso 3 tipos de brocas: diamantada, carbide de tungstênio e brocas de acabamento de carbide e tungstênio. Assim, 105 dentes humanos foram preparados para receber uma coroa metalocerâmica e avaliados através de microscópio eletrônico. A rugosidade superficial foi $8.6(\mathrm{Ra})$ para preparos realizados com brocas diamantadas e $6.8(\mathrm{Ra})$ para brocas carbide de tungustênio. Preparos realizados com brocas de acabamento apresentaram rugosidade superficial maior que 1 .

Pegoraro $^{23}$ (1998) afirmou que as PPFs devem seguir, por exemplo, o princípio mecânico da retenção, que impede o deslocamento axial da restauração quando ela é submetida a uma carga de traçáo, ou seja, o preparo deve apresentar uma retenção friccional que é o contato entre as superfícies internas da restauraçáo e a superfície externa do dente, assim, quanto mais paralelas às paredes axiais do dente preparado, maior vai ser a retençáo friccional. Porém, é importante lembrar que a retenção friccional exagerada pode dificultar a cimentação, devido à resistência de escoamento do cimento, impedindo o assentamento final da prótese, causando o desajuste oclusal e cervical da coroa. Em casos de dentes longos, como ocorre após, por exemplo, tratamento periodontal, pode aumentar a inclinação das paredes para uma convergência oclusal de $10^{\circ}$. Por outro lado, coroas curtas devem apresentar paredes com inclinação próxima ao paralelismo e devem receber meios adicionais de retenção, como confecção de sulcos ou caixas com o intuito de aumentar a área de superfície de contato.

Luk e Tsai ${ }^{19}$ (1996) afirmaram que o deslocamento da coroa deve ser evitado pela forma de resistência da restauraçáo. Assim, quando ocorre a incidência de uma força lateral, devido ao ciclo mastigatório ou hábitos parafuncionais, a restauração tende a girar em torno de um fulcro cujo raio forma um arco tangente na parede oposta do preparo, deixando o cimento sujeito às forças de cisalhamento, que pode provocar trincas no material de cimentação.

Goodacre et al. ${ }^{13}$ (2001) e Miller ${ }^{21}$ (1977) afirmaram que o preparo deve ser executado de tal forma que a restauração apresente espessura suficiente de metal (para coroas totais metálicas), metal e porcelana (para coroas metalocerâmicas) ou porcelana (para coroas metal free), provendo, assim, rigidez estrutural para que as restauraçóes possam resistir a forças mastigatórias sem comprometer a estética e o tecido periodontal.

Gardener $^{12}$ (1982) e Ayad et al. ${ }^{2}$ (1996) afirmaram que os principais objetivos no tratamento com próteses fixas é a preservação da saúde periodontal e pulpar. Assim, o calor gerado durante o preparo, a qualidade das brocas, a quantidade de dentina remanescente e a reação exotérmicas dos materiais empregados podem provocar danos pulpares, aumentando assim o tempo de tratamento e os custos. Da mesma forma, a preservação da saúde periodontal também se torna importante; assim, estão diretamente relacionados a esse objetivo: a higiene oral, forma, contorno e localização da margem cervical do preparo, que pode ser supragengival, subgenvival ou ao nível do término gengival. De acordo com Bowley et al. ${ }^{5}$ (2004) a melhor localização do término cervical é aquela em que o profissional pode controlar todos os procedimentos clínicos e o paciente tem condição de realizar uma adequada higienização.

\section{Preparos dentais para PPRs}

Em relação aos preparos dentais em próteses parciais removíveis, Rudd et al. ${ }^{30}$ (1999) afirmaram que as primeiras publicaçóes datam de 1711, mas o delineador foi desenvolvido apenas em 1915 e comercializado a partir de 1921. Porém, somente em 1940 com as publicaçóes de Applegate sobre o conceito de paralelismo, a PPR passou a ter os moldes de hoje.

Para que uma PPR possa alcançar seus objetivos no sentido de restaurar a função, a estética, a preservação dos dentes remanescentes e seus tecidos de suporte, a boca 
terá de ser preparada convenientemente para receber o aparelho protético. Esse preparo da boca do paciente de PPR consiste basicamente em restabelecer a condição de saúde e melhorar a biostática dos dentes remanescentes, com finalidade de proporcionar uma melhor condição biomecânica (Dykema et al. $\left.{ }^{10}, 1969\right)$.

Steward et al. ${ }^{38}$ (1992) afirmaram que se quando um dente é perdido, seus adjacentes tendem a inclinar em direção ao espaço criado. $\mathrm{O}$ mesmo ocorre quando seu antagonista é perdido, provocando a extrusão do elemento dentário. Quando o plano oclusal não está em harmonia com o arco dental, reposicionar dentes artificiais criando uma função harmoniosa pode se tornar um grande desafio. Becker et al. ${ }^{3}$ (1994) afirmaram que o nivelamento do plano oclusal tem por objetivo restabelecer a posição de todos os dentes remanescentes na direção ântero-posterior, tomando-se como referência os segmentos restantes do plano oclusal original, porventura ainda presentes na dentição remanescente. Isso poderia ser realizado da seguinte maneira: por redução dos dentes que ultrapassam o plano oclusal em um grau mínimo e, nesse caso, o desgaste se limitará à capa de esmalte; grau médio impóe a necessidade da instalação de algum material restaurador; já o grau máximo, acontece quando os dentes ultrapassam exageradamente o plano oclusal de referência, sendo inevitável o envolvimento não só da dentina, mas também do órgão pulpar, obrigando a realização de endodontia, caso em que a restauração ocorre por meio de coroas totais. $\mathrm{O}$ nivelamento do plano oclusal também pode ocorrer por acréscimos, nos dentes que não alcançam o plano oclusal.

Bridgeman et al. ${ }^{5}$ (1997) e Ahmad et al. ${ }^{1}$ (1992) afirmaram que a melhora das condiçôes biostáticas tem por objetivo propiciar aos dentes suportes melhores condiçôes para receberem as cargas que irão obrigatoriamente incidir sobre eles. $\mathrm{O}$ que se pretende é que essa carga seja o mais axial possível. Assim, os planos-guias são determinados nas faces axi-proximais dos dentes suportes, por desgaste da convexidade anatômica, permitindo que o aparelho protético deslize de maneira suave, guiado pelos diversos planos. Os objetivos dos planos-guias seriam: criar uma única via de inserção e remoção da prótese e evitar a impacção alimentar. Deve ser localizado entre o $1 / 3$ médio e gengival da proximal, apresentar uma largura V-L de 1 a $1,5 \mathrm{~mm}$ e profundidade de $0,5 \mathrm{~mm}$.

Weintraub $^{40}$ (1985), Rudd et al. ${ }^{29}$ (1986) e Todes$\operatorname{can}^{39}$ (1996) afirmaram que o braço de oposição deve ter seu limite no $1 / 3$ médio e gengival da face lingual. Esse deve ser necessariamente rígido para atender aos princípios biomecânicos da reciprocidade. $\mathrm{O}$ que equivale dizer que o braço de oposição deve ficar posicionado na face expulsiva da face palatina ou lingual do dente suporte. Assim, torna-se necessária a realização de desgastes para adequar a linha equatorial. Rudd et al. ${ }^{29}$ (1986) e Todescan ${ }^{39}$ (1996) também afirmaram que a ponta ativa do grampo de retenção deve ficar no limite do $1 / 3$ médio e gengival. Em alguns casos, porém, a calibragem da retenção poderá impor a necessidade de algum desgaste na área, caso haja retenção excessiva. Caracteriza-se por retençáo excessiva a situaçáo em que o limite do $1 / 3$ gengival apresenta na calibragem uma retenção superior a determinada para cada tipo de liga do grampo. Para o cobalto-cromo, por exemplo, a retenção deve ser de 0,25. Admitindo-se que uma convexidade acentuada no dente tenha resultado numa retenção de 0,5 ou 0,75 no limite do $1 / 3$ médio e gengival, impóe-se a necessidade de desgaste dessa convexidade para que se alcance a retenção desejada.

Os apoios foram inicialmente estudados por Bonwill em 1899, que os considerou como peças fundamentais no bom funcionamento dos grampos (Wong et al. ${ }^{42}$, 1993). O preparo para os descansos oclusais tem objetivo de prevenir a possibilidade de sua interferência com os dentes do arco oponente. As principais funçóes dos apoios seriam manter a relaçáo oclusal; prover estabilidade da prótese; prevenir lesão de tecido mole e garantir correta relação dos elementos do grampo (Rudd et al. ${ }^{29}$, 1986). De acordo com Todescan ${ }^{39}$ (1996), os apoios podem ser classificados em primários, aqueles adjacentes, e secundários os distantes do espaço protético; intracoronários ou extracoronários, quando estiverem respectivamente dentro ou fora dos limites anatômicos da coroa; e, ainda, os superficiais ou profundos. Podendo ainda, ser posicionados na crista marginal da oclusal do dente suporte, no cíngulo ou incisal.

\section{Preparo dental para Prótese Hibrida}

Já a prótese total híbrida foi inicialmente descrita por Ledger em 1856 e pode ser definida como uma prótese dentomucossuportada que pode se apoiar em um ou mais dentes ou raízes suportes. Apresenta a vantagem de preservar a estrutura óssea, devido à manutenção dos remanescentes radiculares, além de ser uma prótese em que o paciente apresenta maior força mastigatória, maior eficiência mastigatória e padrôes de mastigação mais consistentes (Weintraub ${ }^{41}, 1987$ ). 
A prótese híbrida prepara o paciente psicologicamente para o edentulismo e o uso de prótese total convencional. Reitz ${ }^{26}$ (1980) realizou um trabalho com pacientes dentados, usuários de PT convencional, e pacientes usuários de PT híbrida. $\mathrm{O}$ autor concluiu que pacientes dentados apresentaram uma eficiência mastigatória de 90\%, usuários de PT convencional 59\% e usuários de PT híbrida uma eficiência de $70 \%$.

A seleção do pilar para a prótese total híbrida é ditada pelo número de dentes que restaram, pela posição destes nos arcos, pela situação endodôntica, presença de lesão, comprimento e largura da raiz. Perel ${ }^{24}$ (1973) realizou um trabalho com o objetivo de mensurar a reabsorção óssea, através de radiografias cefalométricas, em pacientes usuários de PT convencional e prótese híbrida. Assim, foram obtidos 2 grupos: o 1oㅡ, constituído por usuários de PT superior e prótese híbrida inferior; e o 2o, usuários de PT dupla. Os autores observaram que pacientes usuários de prótese híbrida apresentaram uma reabsorção de apenas $0,6 \mathrm{~mm}$, contra $5,2 \mathrm{~mm}$ de reabsorção dos pacientes usuários de PT convencional.

Num estudo longitudinal, Rissim ${ }^{28}$ (1978) realizou um trabalho sobre eficiência mastigatória, e concluiu que pacientes usuários de prótese híbrida apresentaram uma eficiência mastigatória de 50\% maior que pacientes usuários de PT convencional.

De acordo com Weintraub ${ }^{41}$ (1987), a prótese híbrida apresenta algumas desvantagens, tais como o histórico do paciente, que já perdeu todos os dentes, por doença periodontal ou cárie. Além disso, a manutenção de raízes e a instalação de processos inflamatórios, devidos à cárie ou doença periodontal, podem levar à perda de mais osso do que a simples extraçáo.

\section{DISCUSSÃO}

O sucesso de uma reabilitação deve levar em consideração três critérios: a longevidade da prótese, a saúde bucal e a satisfação do paciente. Para alcançar tais objetivos, o cirurgião-dentista deve saber executar todas as fases do procedimento. De nada adianta um dente estar bem preparado se o profissional negligenciar, por exemplo, as moldagens. O sucesso do tratamento pode ser comparado a uma corrente, a ruptura de um elo dessa corrente, destrói, e acaba com a corrente. Com o preparo de dentes ocorre a mesma coisa: como a prótese apresentará longevidade, se o dentes preparados não apresentarem condiçôes mecânicas para mantê-la em posição, ou se o desgaste foi exagerado e alterou a biologia da polpa, se o término cervical foi levado muito subgengivalmente quebrando a homeostasia da área, ou se a estética foi prejudicada devido um desgaste insuficiente gerando um sobrecontorno. Para isso, um preparo dental não deve ser iniciado sem que o profissional conheça os princípios fundamentais para conseguir preparos corretos (Pegora$\left.\mathrm{ro}^{23}, 1998\right)$.

Seymour et al. ${ }^{32}$ (1996) afirmaram que as coroas metaloceramicas são as mais populares e que seu fracasso está geralmente relacionado à fratura ou estética, devido à realização de preparos dentais inadequados. Por isso, os autores realizaram um estudo em que avaliaram através de escaneamento, 24 dentes humanos extraídos e preparados para receberem esse tipo de coroa. Os autores observaram uma deficiência no preparo dos términos cervicais principalmente no que diz respeito à espessura que pode provocar implicaçóes, tais como, longevidade do trabalho protético ou problemas estéticos e periodontais.

Oyar et al. ${ }^{22}$ (2006) realizaram um trabalho para avaliar a transmissão de forças através de elementos finitos, em 2 tipos de preparos dentais: o 1o, um preparo anatômico para coroas metalocerâmicas, no qual o desgaste segue a anatomia do pré-molar preparado; e o 2o, que é a simulação de um preparo não anatômico, como se o dente fosse preparado em formato de um cilindro. Os autores concluíram que o preparo anatômico apresentou melhor distribuição de forças na estrutura dental, e que o preparo não anatômico apresentou melhor distribuição de forças na estrutura da coroa metalocerâmica.

Rudd et al. ${ }^{30}$ (1999) realizaram um trabalho sobre o preparo dental em prótese parcial removível. Os autores ressaltaram que o sucesso ou o fracasso da PPR depende de alguns fatores, e que, para se alcançar o sucesso o profissional, deve-se desenvolver uma sequência de etapas de preparos dentais, obtidos a partir do plano de tratamento, que deve ser baseado em evidências clínicas e radiográficas. $\mathrm{O}$ autor também ressalta a importância do preparo de boca, de planos-guias e dos nichos oclusais para os apoios, afirmando que o adequado preparo desses desgastes pode diminuir e muito os riscos de fracassos nos tratamentos com PPR.

Bezzon et al. ${ }^{4}$ (1997) salientaram a importância em se determinar uma única via de inserção e remoção obtida a partir da confecção de planos-guias. Inclusive, Schwarz e Barsby ${ }^{31}$ (1984) destacaram a importância da confecção dos planos-guias, que têm por objetivo minimizar a transmissão de estresse para o dente suporte da PPR; 
facilitar a inserção e a remoção da prótese pelo paciente; evitar a impacção alimentar, por reduzir o espaço presente entre o dente e a PPR; contribuir na retenção da prótese; melhorar a estabilidade da prótese, reduzindo o deslocamento desta devido à incidência de forças horizontais.

Rudd et al. ${ }^{29}$ (1986) afirmaram que muitas próteses parciais removíveis não apresentam preparos dentais essenciais para a correta função. Muitas até se apresentam esteticamente aceitáveis, porém, geralmente com problemas na estabilidade e retenção. Uma análise crítica, por parte dos autores, levou-os a afirmar que a insatisfação dos pacientes pode ser evitada com a diminuição do deslocamento da prótese durante a fala e mastigação, ou seja, requisito básico para o bom funcionamento da prótese e satisfação dos pacientes é o preparo adequado dos dentes-suportes da PPR.

Weintraub $^{41}$ (1987) afirmou que existe um grande segmento da população que necessita de reabilitação completa na cavidade bucal, pacientes estes cuja reabilitação difere drasticamente dos tratamentos a partir de PPF ou PPR. Esse segmento da população, geralmente são pacientes geriátricos, que apresentam poucos remanescentes dentários em ambos os arcos, ou, às vezes, com edentulismo na maxila e poucos remanescentes na mandíbula. Brill ${ }^{6}$ (1958) definiu inicialmente esse tipo de reabilitação como sendo prótese híbrida, que seria uma prótese que apresenta algumas características de PPR associada a outras características de PT. Outros termos também podem ser utilizados para denominar esse tipo de prótese, tais como, overdentures, prótese telescópica, prótese total retida por dentes, prótese biológica e prótese overlay.

Kelly e Nakamoto ${ }^{17}$ (1974) destacaram as indicaçóes para as próteses híbridas, que poderiam ser confeccionadas em pacientes, com poucos remanescentes dentários ou radiculares; pacientes apresentando situaçóes em que PPRs ou PPFs, não podem ser confeccionadas, como em casos de problemas congênitos; pacientes com prognóstico ruim para a instalação de uma prótese total con- vencional, tais como, com atrofia de rebordo alveolar; e em casos de pacientes que não estão psicologicamente preparados para se tornarem desdentados totais. Nesse caso a prótese híbrida funcionaria como uma prótese intermediária, que seria utilizada antes da instalação da prótese total convencional.

As vantagens inerentes a esse tipo de prótese seriam o aumento da estabilidade e retençáo; melhor controle do profissional no desenvolvimento de um esquema oclusal para a prótese; a conversão simples da prótese híbrida, caso haja a necessidade, em prótese total convencional; melhor registro das posiçóes intermaxilar devido à presença dos remanescentes dentários; e o custo, que é bem inferior à combinação de prótese fixa com prótese removível (Weintraub ${ }^{41}$, 1987).

Assim, o preparo dental para uma prótese é um dos fatores críticos que deve ser cuidadosamente planejado e executado para permitir a longevidade do tratamento que deve restabelecer função mastigatória e fonética, além de estética, requisitos esses, fundamentais para a satisfação do paciente perante os resultados finais de uma reabilitação.

\section{CONCLUSÃO}

Diante dos trabalhos apresentados, nesta revisão de literatura, pode-se concluir que:

O sucesso de uma reabilitaçáo deve levar em consideração a longevidade da prótese, a saúde bucal e a satisfação do paciente. No caso de próteses fixas, o objetivo é preservar a biologia pulpar e periodontal, sem prejudicar a estética, realizando-se desgastes adequados para prevenir sobrecontornos. $\mathrm{Na}$ prótese parcial removível, o principal objetivo é alcançar uma adequada biostática nos dentes-suporte, para prevenir sobrecargas, além de melhorar a retenção e a estabilidade da prótese, tornando-a fácil de ser inserida ou removida pelo paciente. Os preparos dentais nas próteses híbridas devem permitir fácil higienização e integridade dos remanescentes dentários, para que apresentem longevidade, garantindo-se o sucesso desse tipo de prótese. 


\section{REFERÊNCIAS}

1. Ahmad L, Sherriff M, Waters NE. The effect of reducing the number of claps on removable partial denture retention. J Prosthet Dent 1992 Dec; 68(6): 928-33.

2. Ayad MF, Rosenstiel SF, Hassan MM. Surface roughness of dentin after tooth preparation with different rotary instrumentation. J Prosthet Dent 1996 Feb; 75(2): 122-128.

3. Becker CM, Kaiser DA, Goldfogel MH. Evolution of removable partial denture design. J Prosthod 1994 Sep; 3(3): 158-66.

4. Bezzon OL, Mattos MG, Ribeiro RF. Surveying removable partial dentures: The importance of guiding planes and path of insertion for stability. J Prosthet Dent 1997 Oct; 78(4): 412-18.

5. Bowley JF, Sun AF, Barouch KK. Effect of margin location on crown preparation resistence form. $J$ Prosthet Dent 2004 Dec; 92(6): 546-550.

6. Bridgeman JT, Marler VA, Hummel SK, Benson BW, Pacel LL. Comparison of titanium and cobaltchromium removable partial denture clasps. J Prosthet Dent 1997 Aug; 78(2): 187-93.

7. Brill N. Adaptation and the hybrid prosthesis. J Prosthet Dent 1955 Nov; 5(6): 811-24.

8. Chandler NP. Etched metal prostheses to retain severely traumatized teeth in function. Endod Dent Traumatol 1989 Apr; 5(2): 105:8.

9. Cheung GS, Dimmer A, Mellor R, Gale M. A clinical evaluation of conventional bridgework. J Oral Rehab 1990 May; 17(2): 131-6.

10. Dykema RW, Cuningham DM, Johnston JF. Modern practice in removable partial prosthodontics. 1o ed Philadelphia: WB Saunders Co; 1969.417p.

11. El-Ebrashi MK. Craig RG, Peyton FA. Experimental stress analysis of dental restorations. VI. The conception of proximal reduction in complain restoration. J Prosthet Dent 1969 Dec; 22(6): 663-70.

12. Gardner MF. Margins of complete crowns - Literature review. J Prosthet Dent 1982 Oct; 48(4): 396400.
13. Goodacre CJ, Campagni Wv, Aquilino AS. Tooth preparations for complete crowns: an art form based on scientific principles. J Prosthet Dent 2001 Apr; 85(4): 363-76.

14. Guyer SE. Multiple preparations for fixed prosthodontics. J Prosthet Dent 1970 May; 23(5): 529-553.

15. Guyer SP, Lefkowitz W, Malone WFP, Rhoads JE, Sproule RC. An analysis of margin configuration for metal-ceramic crows. J Prosthet Dent 1985 Feb; 53(2): 153-7.

16. Hobo S, Shillingburg HT. Porcelain fused to metal: tooth preparation and coping design. J Prosthet Dent 1973 Jul; 30(1): 28-36.

17. Kelly E, Nakamoto Ry. Cleidocranial dysostosis. a prosthodontic problem. J Prosthet Dent 1974 May; 31(5): 518-26.

18. Lorato DC. The effect of crown margin extension on gingival inflammation. J South Calif Dent Assoc 1969 Nov; 37(11): 476-8.

19. Luk K, Tsai T. Improved resistance and retention of a short coronal tooth preparation for a complete crown. J Prosthet Dent 1996 Mar; 75(3): 340.

20. Miller IF, Belsky MW. The full shoulder preparation for periodontal health. Dent Clin North Am 1965 May; 23: 83-102.

21. Miller LL. Framework design in ceramo-metal restorations. Dent Clin North Am 1977 Oct; 21(4): 699716.

22. Oyar P, Ulusoy M, Eskitascioglu G. Finite element analysis of stress distribution of 2 different tooth preparation design in porcelain-fused-to-metal crowns. Int J Prosthdont 2006 Jan-Feb; 19(1): 85-9.

23. Pegoraro LF. Prótese fixa 1998 São Paulo: Artes Médicas, 1998.313p.

24. Perel M. Telescope denture. J Prosthet Dent 1973 Feb; 29(21): 151-6.

25. Preston J. Rational approach to tooth preparation for ceramometal restorations. Dent Clin North Am 1977 Oct; 21(4): 683-87. 
26. Reitz P, Weiner MG, Levin B. An overdenture survey, second report. J Prosthet Dent 1980 Apr; 43(4): 457-62.

27. Richter WA, Ueno H. Relationship of crown margin placement to gingival inflammation. J Prosthet Dent 1973 Aug; 30(2): 156-61.

28. Rissin L, House JE, Manly RS, Kapur KK.Clinical comparison of masticatiry performance and electromyographic activity of patients with complete dentures, overdentures and natural teeth. J Prosthet Dent 1978 May; 39(5): 508-11.

29. Rudd KD, Morrow RM. Rhoads JE. Dental laboratory procedures for removable partial dentures. 2oed St Louis: CV Mosby; 1986. p.164-86.

30. Rudd RW, Bange AA, Rudd KD, Montalvo R. Preparing teeth to receive a removable partial denture. $J$ Prosthet Dent 1999 Nov;82(5):536-49.

31. Schwarz WD, Barsby MJ. Tooth alteration procedures prior to partial denture construction Part 3. Dent Update 1984 May; 11(4): 231-2,234-7.

32. Seymour K, Zou L, Samarawickrama DYD, Lynch E. Assessment of shoulder dimensions and angles of porcelain bonded to metal crown preparations. $J$ Prosthet Dent 1996 Apr; 75(4): 406-11.

33. Silness J. Distribution of artificial crowns and fixed partial dentures. J Prosthet Dent 1970 Jun; 23(6): 641-7.

34. Shillingburg HT, Hobo S. Fundamentos de prótese fixa. São Paulo: Melhoramentos,1997.
35. Smith PW, Wilson NH. Shade selection for singleunit anterior metal ceramic crowns: a 5-year retrospective study of 2,500 cases. Int J Prosthodont 1998 Jul-Aug; 11(4): 302-6.

36. Sozio RB. The marginal aspect of ceramo-metal restoration. the collarless ceramo-metal restoration. Dent Clin North Am 1977 Oct; 21(4): 787-801.

37. Stein RS, Glickman I. Prosthetic considerations essential for gingival health. Dent Clin North Am 1960 Mar; 4(2): 177-88.

38. Steward KL, Rudd KD, Kuebker WA. Clinical removable partial prosthodontics. $2^{\text {(ed) }}$. St Louis: Ishiyaku EuroAmerica Inc., 1992. p.221-7.

39. Todescan R. Atlas de prótese parcial removível. São Paulo: Santos, 1996.

40. Weintraub GS. Review or removable partial denture components and their design as related to maintenance of tissue health. Dent Clin North Am 1985 Jan; 29(1): 39-56.

41. Weintraub GS. Hybrid prosthetic dentistry. Dental Clin North Am 1987 Jul; 31(3): 441-56.

42. Wong MT, Calverley MJ, Nagy WW. Removable partial denture framework try-in. J Prosthet Dent 1993 Apr; 69(4): 363-8.

Recebido em: 12/9/2007 Aceito em: 5/12/2007 